\title{
Circulation Journal Awards for the Year 2020
}

\author{
Toshihisa Anzai, MD, PhD
}

Dear Colleagues,

On behalf of the Editorial Team of Circulation Journal, I am pleased to announce the Circulation Journal Awards for the Year 2020.

The aim of these Awards is to recognize papers published in 2020, both clinical and experimental studies, that were highly appreciated by the Editorial Team. The selection process comprises 2 steps. In the first step, from 203 original papers published in the Journal in 2020, our 41 Japanese Associate Editors selected papers with a high scientific level in their respective fields, and in the second step, the 4 Associate Editorial Teams (10 11 on 1 team) further evaluated the selected papers in terms of originality, contribution to cardiovascular science, manner of paper preparation, and future possibilities.

In the year of 2020, the following 7 papers have been selected for the Circulation Journal Awards.

First Place in the Clinical Investigation Section

(Circ J 2020; 84: 769-775)1

\section{Atherosclerotic Coronary Plaque Is Associated With Adventitial Vasa Vasorum and Local Inflammation in Adjacent Epicardial Adipose Tissue in Fresh Cadavers}

Hiroyuki Ito*, Tetsuzo Wakatsuki, Koji Yamaguchi, Daiju Fukuda, Yutaka Kawabata, Tomomi Matsuura, Kenya Kusunose, Takayuki Ise, Takeshi Tobiume, Shusuke Yagi, Hirotsugu Yamada, Takeshi Soeki, Yoshihiro Tsuruo, Masataka Sata

(Department of Cardiovascular Medicine (H.I., T.W., K. Y., Y.K., T.M., K.K., T.I., T.T., S. Y., T.S., M.S. ), Department of Cardio-Diabetes Medicine (D.F.), Department of Community Medicine for Cardiology (H.Y.), Department of Anatomy and Cell Biology (Y.T.), Tokushima University Graduate School of Biomedical Sciences, Tokushima, Japan)

* Current affiliation: Department of Community Medicine for Cardiology, Tokushima University Graduate School of Biomedical Sciences, Tokushima, Japan

Background: The coronary adventitia has recently attracted attention as a source of inflammation because it harbors nutrient blood vessels, termed the vasa vasorum (VV). This study assessed the link between local inflammation in adjacent epicardial adipose tissue (EAT) and coronary arterial atherosclerosis in fresh cadavers.

Methods and Results: Lesion characteristics in the left anterior descending coronary artery of 10 fresh cadaveric hearts were evaluated using integrated backscatter intravascular ultrasound (IB-IVUS), and the density of the $\mathrm{VV}$ and levels of inflammatory molecules from the adjacent EAT were measured for each of the assessed lesions. The lesions were divided into lipid-rich, lipid-moderate, and lipid-poor groups according to percentage lipid volume assessed by IB-IVUS. Higher expression of inflammatory molecules (i.e., vascular endothelial growth factor A $[V E G F A]$ and $V E G F B)$ was observed in adjacent EAT of lipid-rich $(\mathrm{n}=11)$ than in lipid-poor $(\mathrm{n}=11)$

J-STAGE Advance Publication released online February 16, 2021

Department of Cardiovascular Medicine, Hokkaido University Graduate School of Medicine, Sapporo, Japan

Mailing address: Toshihisa Anzai, MD, PhD, Department of Cardiovascular Medicine, Hokkaido University Graduate School of Medicine, Kita-15 Nishi-7, Kita-ku, Sapporo 060-8638, Japan. E-mail: anzai@med.hokudai.ac.jp

All rights are reserved to the Japanese Circulation Society. For permissions, please e-mail: cj@j-circ.or.jp ISSN-1346-9843 
lesions (7.99 \pm 3.37 vs. $0.45 \pm 0.85$ arbitrary units [AU], respectively, for $V E G F A ; 0.27 \pm 0.15$ vs. $0.11 \pm 0.07 \mathrm{AU}$, respectively, for $V E G F B ; \mathrm{P}<0.05)$. The density of adventitial VV was greater in lipid-rich than lipid-poor lesions $(1.50 \pm 0.58 \%$ vs. $0.88 \pm 0.23 \% ; \mathrm{P}<0.05)$.

Conclusions: Lipid-rich coronary plaques are associated with adventitial VV and local inflammation in adjacent EAT in fresh cadavers. This study suggests that local inflammation of EAT is associated with coronary plaque progression via the VV.

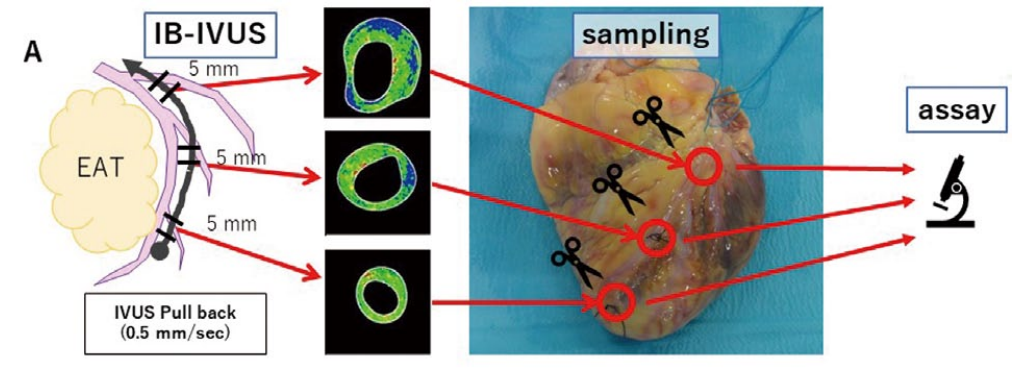

B

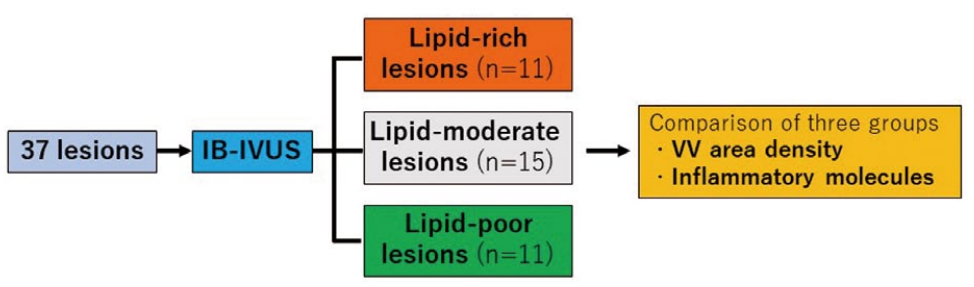

Figure 1. (A) Study design for using integrated backscatter intravascular ultrasound (IB-IVUS) and measuring the adventitial vasa vasorum (VV) area density and inflammatory molecules in adjacent epicardial adipose tissue (EAT) of cadaveric hearts. (B) Lesion classification. In all, 37 lesions were divided into 3 groups (lipid-rich, lipid-moderate, and lipid-poor groups) to measure the VV area density and inflammatory molecules.
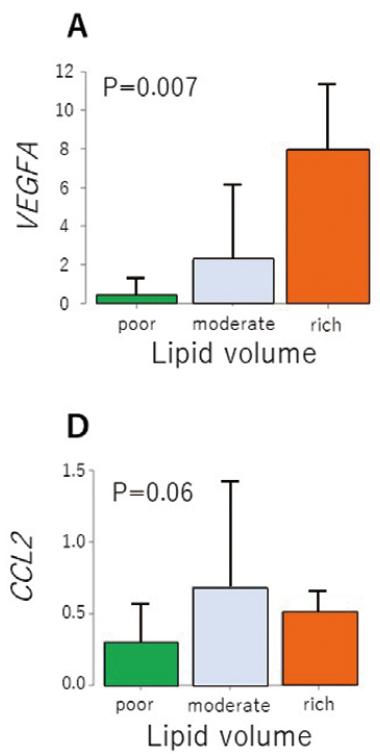
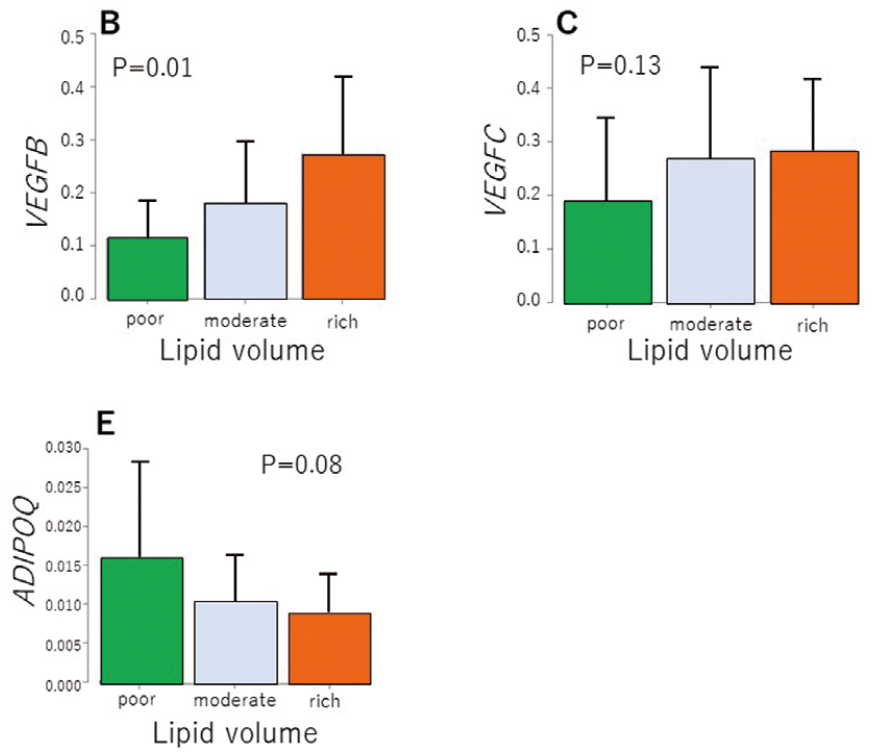

Figure 4. Expression of inflammatory molecules in adjacent epicardial adipose tissue (EAT). (A) Vascular endothelial growth factor A (VEGFA), (B) VEGFB, (C) VEGFC, (D) C-C motif chemokine ligand 2 (CCL2), and (E) adipocyte-specific protein $(A D I P O Q)$ expression was compared among the 3 groups (lipid-rich, lipid-moderate, and lipid-poor groups). P-values are for comparisons between lipid-poor and lipid-rich lesions. There were no significant differences in the expression of inflammatory molecules in adjacent EAT between lipid-moderate lesions and other types of lesions. 


\section{Effect of Therapeutic Modification on Outcomes in Heart Transplantation Over the Past Two Decades - A Single-Center Experience in Japan -}

Masanobu Yanase, Keiichiro Iwasaki, Takuya Watanabe, Osamu Seguchi, Seiko Nakajima, Kensuke Kuroda, Hiroki Mochizuki, Sachi Matsuda, Hiromi Takenaka, Megumi Ikura, Naoki Tadokoro, Satsuki Fukushima, Tomoyuki Fujita, Hatsue Ishibashi-Ueda, Takeshi Nakatani, Soichiro Kitamura, Junjiro Kobayashi, Kenichi Tsujita, Hisao Ogawa, Norihide Fukushima

(Department of Transplant Medicine (M.Y., K.I., T.W., O.S., S.N., K.K., H.M., N.F.), Department of Pharmacy (S.M., H.T., M.I.), Department of Cardiovascular Surgery (N.T., S.F, T.F., S.K., J.K.), Department of Pathology (H.I.-U.), National Cerebral and

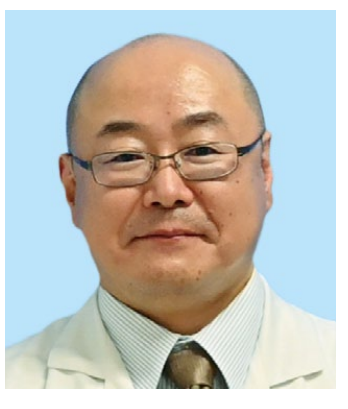
Cardiovascular Center, Suita; Division of Advanced Cardiovascular Medicine (M. Y., N.F.), Department of Cardiovascular Medicine (K.T.), Graduate School of Medical Sciences, Kumamoto University, Kumamoto; Department of Cardiology, Maki Hospital, Osaka (T.N.); and National Cerebral and Cardiovascular Center, Suita (H.O.), Japan)

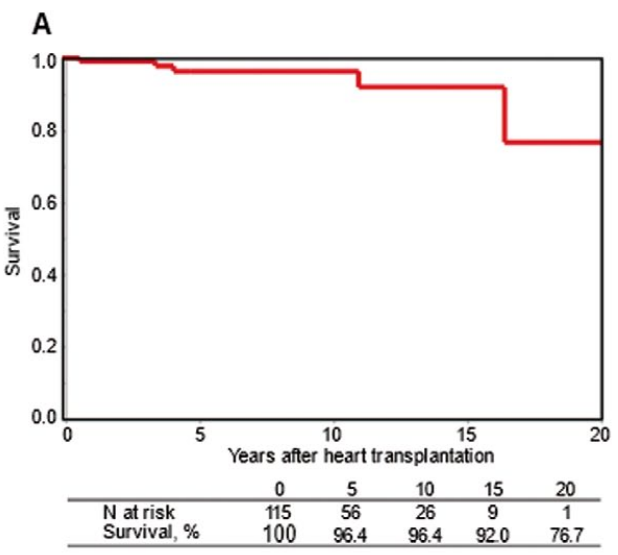

B
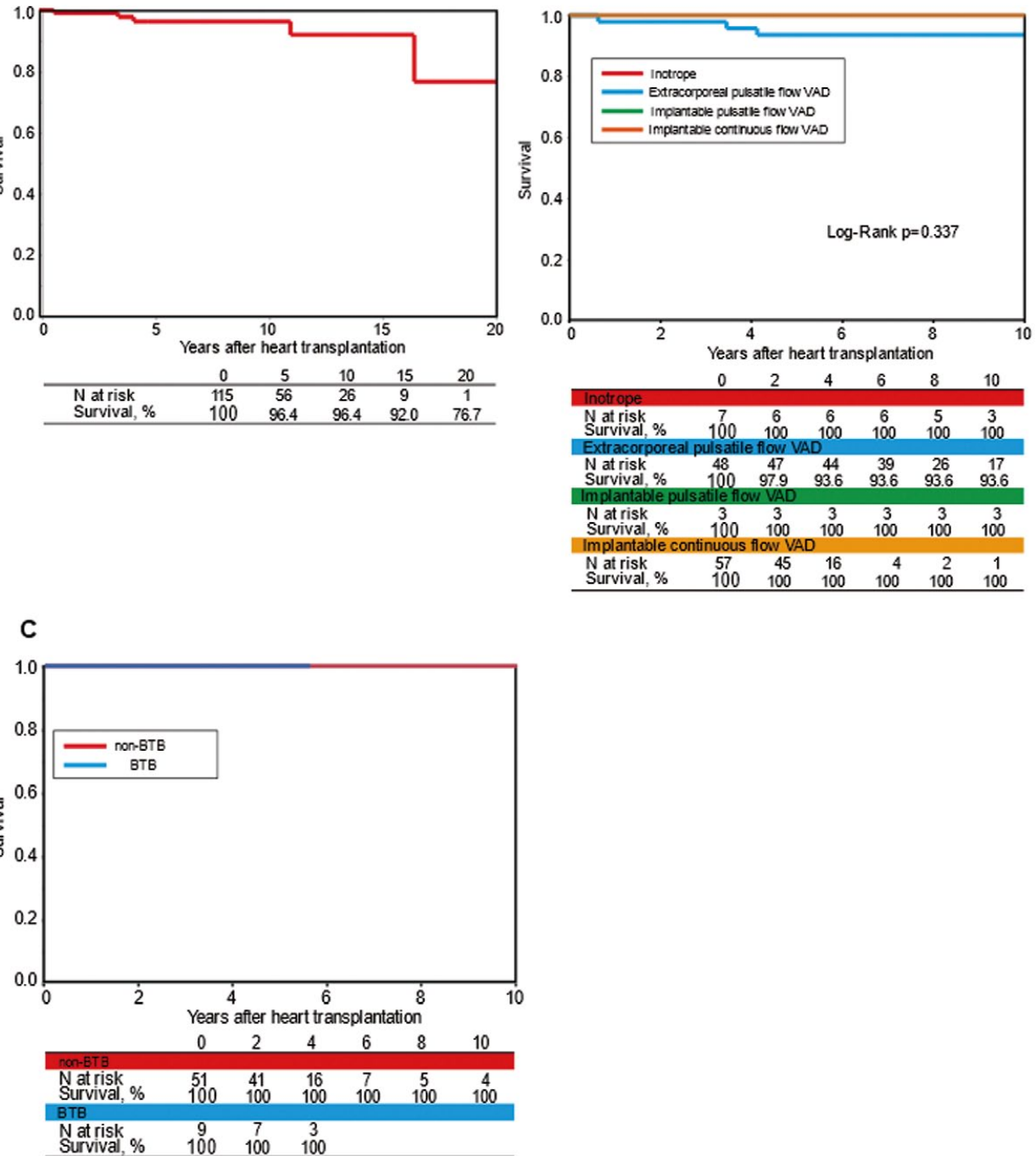

Figure 2. Cumulative patient survival after heart transplantation at the National Cerebral Cardiovascular Center between May 1999 and June 2019. (A) Overall; (B) by pre-transplant recipient condition; (C) by bridge-to-bridge (BTB) status. 
Background: During these 2 decades (1999-2019), many therapeutic strategies have been developed in the field of heart transplant (HTx) to improve post-HTx outcomes. In the present study, 116 consecutive HTx adults between 1999 and 2019 were retrospectively reviewed to evaluate the influences of a therapeutic modification on post HTx outcomes.

Methods and Results: Patient survival, functional status and hemodynamics after HTx and modification of therapeutic strategies were reviewed. The overall cumulative survival rate at 10 and 20 years post-HTx was 96.4 and $76.7 \%$, respectively. There were no significant differences in survival rate or exercise tolerance after HTx between extracorporeal and implantable continuous flow-LVAD. Post-HTx patient survival in patients, irrespective of the donor risk factors such as donor age, low LVEF, history of cardiac arrest, was equivalent across cohorts, while longer TIT and higher inotrope dosage prior to procurement surgery were significant risk factors for survival. In 21 patients given everolimus (EVL) due to renal dysfunction, serum creatinine significantly decreased 1 year after initiation. In 22 patients given EVL due to transplant coronary vasculopathy (TCAV), maximum intimal thickness significantly decreased 3 years after initiation.

Conclusions: The analysis of a 20-year single-center experience with HTx in Japan shows encouraging improved results when several therapeutic modifications were made; for example, proactive use of donor hearts declined by other centers and the use of EVL in patients with renal dysfunction and TCAV.

Second Place in the Clinical Investigation Section

(Circ J 2020; 84: 235-244) 3

\section{Simultaneous Volumetric and Functional Assessment of the Right Ventricle in Hypoplastic Left Heart Syndrome After Fontan Palliation, Utilizing 3-Dimensional Speckle-Tracking Echocardiography}

Tomoyuki Sato*, Renzo JC Calderon, Berthold Klas, Gianni Pedrizzetti, Anirban Banerjee

(Department of Pediatrics, Division of Cardiology, The Children's Hospital of Philadelphia, Philadelphia, PA (T.S., R.J.C.C., A.B. ), USA; TomTec Imaging Systems, Munich (B.K.), Germany; and Department of Engineering and Architecture, University of Trieste, Trieste (G.P.), Italy)

*Current affiliation: Department of Pediatrics, Jichi Medical University Hospital, Tochigi; Department of Pediatrics, International University of Health and Welfare Hospital, Tochigi, Japan
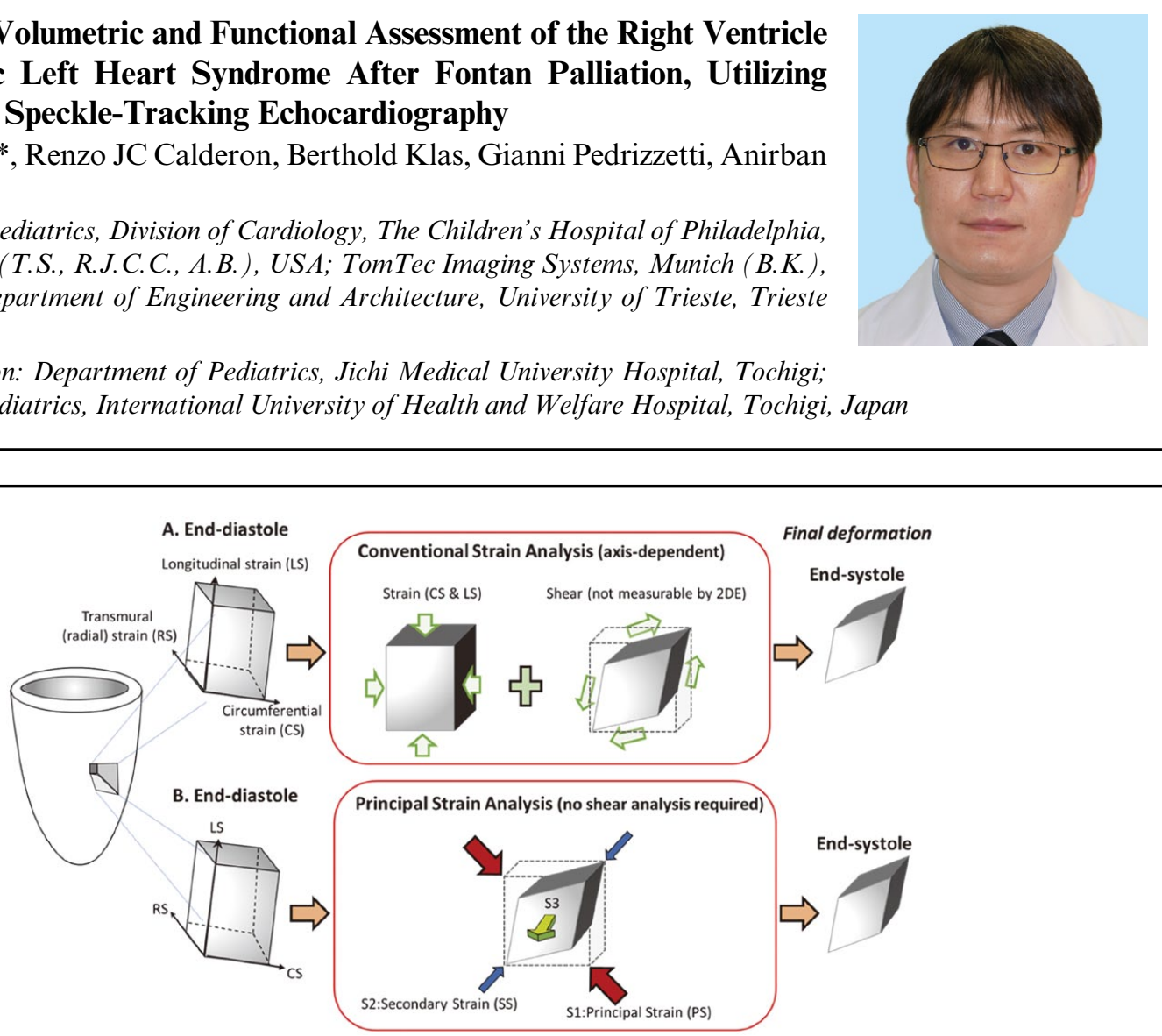

Figure 1. Process of myocardial tissue deformation, showing the 3 orthogonal deformations that completely depict the deformation of a 3D myocardial segment, without the need to consider shear components or the need to limit measurements to conventional axis-dependent directions (longitudinal, circumferential and radial). S1 indicates the PS and is the main contractile force; S2 is the SS, which is transversal to the PS and of much lower intensity; S3 is the positive strain resulting from tissue incompressibility and is mostly represented by thickening of myocardium along the radial direction. The latter cannot be measured by 3D-STE because of the lack of epicardial imaging, but can be measured by cardiac MRI. Moreover, because of tissue incompressibility, S3 is completely dependent on S1 and S2. Therefore, S3 can be excluded and S1 and S2 can provide a simplified, 2-component model of myocardial deformation that is more comprehensive than conventional, axis-dependent strains. 3D, three-dimensional; CS, circumferential strain; LS, longitudinal strain; PS, principal strain; RS, radial strain; SS, secondary strain. 
Background: Right ventricular (RV) volumetric and functional assessments are both crucial for the management of patients with hypoplastic left heart syndrome (HLHS). 3-dimensional echocardiography (3DE) for volume measurements and 2D speckle-tracking echocardiography (2D-STE) for strain analysis are performed separately. 3D-STE is capable of evaluating those parameters simultaneously and providing principal strain (PS), unifying the concepts of myofiber orientation and contraction into a single, maximal contractile direction. However, the application of 3D-STE to HLHS has not been studied and so became the aim of our study.

Methods and Results: 64 HLHS patients after Fontan palliation underwent 3D-STE analysis measuring RV end-diastolic volume index (EDVi), ejection fraction (EF), global PS (GPS), global circumferential strain (GCS), and global longitudinal strain (GLS). Volume measurements were compared between 3D-STE and 3DE, and strains were compared between 3D- and 2D-STE. EDVi and EF showed strong correlations between 3D-STE and $3 \mathrm{DE}$ ( $\mathrm{r}=0.93$ and 0.87 , respectively). GCS and GLS showed moderate correlations between 3D- and 2D-STE $(\mathrm{r}=0.65$ and 0.61 , respectively). GPS showed highest magnitude and excellent correlation with EF ( $\mathrm{r}=-0.95)$. Conclusions: Simultaneous volumetric and functional assessment by 3D-STE was a useful method in this HLHS cohort. PS is a promising parameter for evaluating the RV function of HLHS, which could be useful during longitudinal follow-up.

\section{Association Between Plasma Intact Parathyroid Hormone Levels and the Prevalence of Atrial Fibrillation in Patients With Chronic Kidney Disease - The Fukuoka Kidney Disease Registry Study -}

Hokuto Arase, Shunsuke Yamada, Shigeru Tanaka, Masanori Tokumoto, Kazuhiko Tsuruya, Toshiaki Nakano, Takanari Kitazono

(Department of Medicine and Clinical Science, Graduate School of Medical Sciences, Kyushu University, Fukuoka (H.A., S. Y., S.T., K.T., T.N., T.K.); Department of Internal Medicine, Fukuoka Dental College, Fukuoka (M.T.); and Department of Nephrology, Nara Medical University, Kashihara (K.T.), Japan)

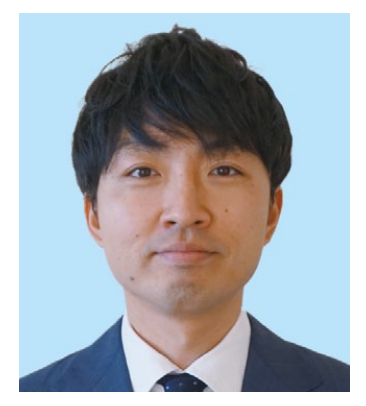

\begin{tabular}{|c|c|c|c|c|c|c|c|c|c|}
\hline & \multicolumn{3}{|c|}{ Unadjusted } & \multicolumn{3}{|c|}{ Age and sex adjusted } & \multicolumn{3}{|c|}{ Multivariable adjusted } \\
\hline & $\begin{array}{c}\text { OR } \\
(95 \% \mathrm{Cl})\end{array}$ & P-value & $\begin{array}{l}P \text { for } \\
\text { trend }\end{array}$ & $\begin{array}{c}\text { OR } \\
(95 \% \mathrm{Cl})\end{array}$ & P-value & $\begin{array}{l}P \text { for } \\
\text { trend }\end{array}$ & $\begin{array}{c}\text { OR } \\
(95 \% \mathrm{Cl})\end{array}$ & P-value & $\begin{array}{l}P \text { for } \\
\text { trend }\end{array}$ \\
\hline Plasma Intact PTH quartile & & & $<0.001$ & & & $<0.001$ & & & 0.016 \\
\hline Q1 (PTH 5-46 pg/mL) & $\begin{array}{l}1.00 \\
\text { (Ref.) }\end{array}$ & - & & $\begin{array}{c}1.00 \\
\text { (Ref.) }\end{array}$ & - & & $\begin{array}{c}1.00 \\
\text { (Ref.) }\end{array}$ & - & \\
\hline Q2 (47-66 pg/mL) & $\begin{array}{c}1.55 \\
(0.90-2.68)\end{array}$ & 0.113 & & $\begin{array}{c}1.38 \\
(0.79-2.40)\end{array}$ & 0.252 & & $\begin{array}{c}1.33 \\
(0.76-2.34)\end{array}$ & 0.321 & \\
\hline Q3 (67-108 pg/mL) & $\begin{array}{c}2.79 \\
(1.69-4.59)\end{array}$ & $<0.001$ & & $\begin{array}{c}2.10 \\
(1.26-3.48)\end{array}$ & 0.004 & & $\begin{array}{c}1.82 \\
(1.06-3.13)\end{array}$ & 0.029 & \\
\hline Q4 (109-1,660pg/mL) & $\begin{array}{c}3.37 \\
(2.07-5.50)\end{array}$ & $<0.001$ & & $\begin{array}{c}2.40 \\
(1.46-3.96)\end{array}$ & $<0.001$ & & $\begin{array}{c}1.99 \\
(1.08-3.64)\end{array}$ & 0.026 & \\
\hline $\begin{array}{l}\text { Per } 50-p g / m L \text { increment } \\
\text { in PTH }\end{array}$ & $\begin{array}{c}1.08 \\
(1.03-1.14)\end{array}$ & 0.007 & & $\begin{array}{c}1.09 \\
(1.03-1.16)\end{array}$ & 0.009 & & $\begin{array}{c}1.09 \\
(1.00-1.20)\end{array}$ & 0.076 & \\
\hline
\end{tabular}

Unadjusted, age- and sex-adjusted, and multivariable-adjusted ORs were analyzed with logistic regression modeling. The covariates included age, sex, SBP, the use of antihypertensive agents and diuretics, diabetes, hyperthyroidism, a history of IHDs, body mass index, smoking status, alcohol intake, hemoglobin, serum albumin, total cholesterol, HDL-C, log[hs-CRP], albumin-corrected calcium and phosphate, eGFR, urinary protein, and FEPi. Two-tailed $\mathrm{P}<0.05$ was considered significant. $\mathrm{CI}$, confidence Intervals; ORs, odds ratios. Other abbreviations as in Table 1.

Background: Parathyroid hormone (PTH) has been associated with cardiovascular disorders; however, it is unknown whether plasma PTH concentrations are associated with atrial fibrillation (AF) in patients with chronic kidney disease (CKD).

Methods and Results: The present cross-sectional study analyzed baseline data of 3,384 patients registered in the Fukuoka Kidney Disease Registry Study, a Japanese multicenter prospective cohort study of patients with non-dialysis-dependent CKD. The outcome was prevalence of AF, and the main risk factor was plasma intact 
PTH concentration. Associations between plasma intact PTH concentration quartiles (Q1-Q4, from lowest to highest) and the presence of AF were analyzed using logistic regression. In all, 185 patients had AF; 22, 34, 59, and 70 patients were in Q1, Q2, Q3, and Q4 of PTH concentrations, respectively. The prevalence of AF increased incrementally with increases in plasma intact PTH. In the logistic regression model, patients with higher plasma intact PTH concentrations (Q2-Q4) had higher adjusted odds ratios ( $95 \%$ confidence intervals) for the prevalence of AF relative to the reference group (Q1), namely 1.33 (0.76-2.34), 1.82 ([1.06-3.13), and 1.99 (1.08-3.64), respectively $(\mathrm{P}=0.016)$.

Conclusions: Higher plasma intact PTH concentrations were significantly and incrementally associated with an increased prevalence of AF in non-dialysis-dependent CKD patients.

\section{Pristane/Hypoxia (PriHx) Mouse as a Novel Model of Pulmonary Hypertension Reflecting Inflammation and Fibrosis}

Hiroyoshi Mori, Tomohiko Ishibashi, Tadakatsu Inagaki, Makoto Okazawa, Takeshi Masaki, Ryotaro Asano, Yusuke Manabe, Keiko Ohta-Ogo, Masashi Narazaki, Hatsue Ishibashi-Ueda, Atsushi Kumanogoh, Yoshikazu Nakaoka

(Department of Vascular Physiology (H.M., T. Ishibashi, T. Inagaki, M.O., T.M., R.A., Y.M., Y.N.), Department of Advanced Medical Research for Pulmonary Hypertension (R.A. ), National Cerebral and Cardiovascular Center Research Institute, Suita; Department of Respiratory Medicine and Clinical Immunology (H.M., Y.M., M.N., A.K.), Department of Cardiovascular Medicine (T.M., Y.N.), Department of Advanced Clinical and Translational

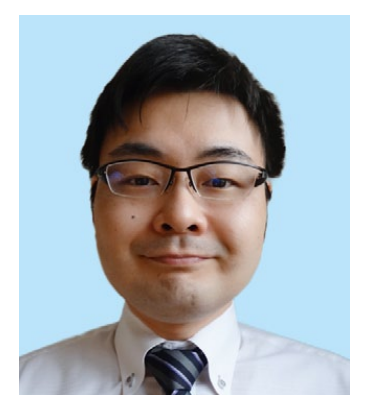
Immunology (M.N.), Osaka University Graduate School of Medicine, Suita; and Department of Pathology, National Cerebral and Cardiovascular Center, Suita (K.O.-O., H.I.-U.), Japan)
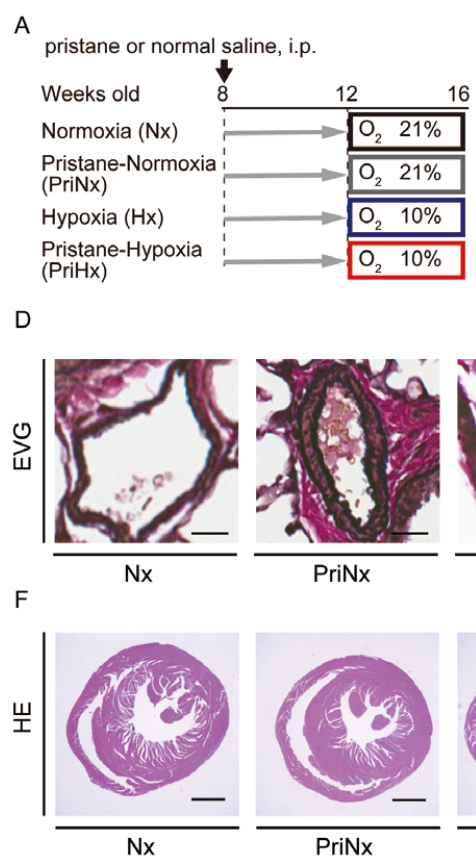
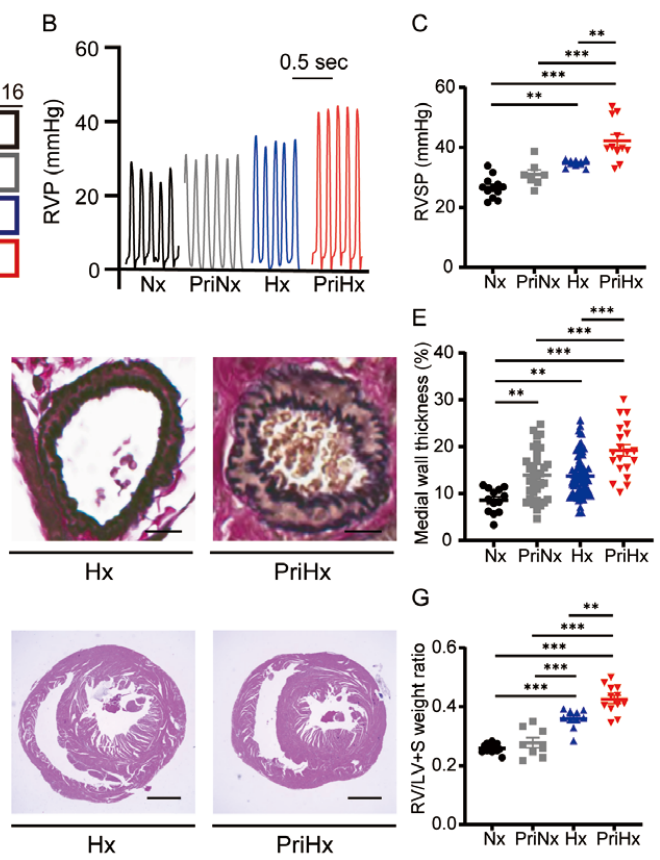

Figure 1. Addition of pristane to chronic hypoxia exacerbates hypoxia-induced pulmonary hypertension (HPH). (A) Experimental protocols and groups. Each mouse was administered $500 \mu \mathrm{L}$ of pristane or normal saline intraperitoneally (i.p.). (B) Representative records of right ventricular pressure. (C) Right ventricular systolic pressure (RVSP) of each group, $\mathrm{n}=7-12$. (D) Representative images of pulmonary arteries with a diameter around $50 \mu \mathrm{m}$ stained with Elastica van Gieson (Scale bars: $10 \mu \mathrm{m}$ ). (E) Medial wall thickness of pulmonary arteries with a diameter $<200 \mu \mathrm{m}$, as the $\mathrm{n}=3$ in each group, which has 13-77 vessels in each. (F) Representative images of right ventricular hypertrophy by hematoxylin and eosin (H\&E) staining. (G) Right ventricle/left ventricle+septum (RV/LV+S) weight ratio, $n=8-12$ in each group. Values shown are the means \pm SEM. One-way analysis of variance was used to detect differences. ${ }^{\star} P<0.05,{ }^{* \star} P<0.01,{ }^{* \star *} P<0.001$. 

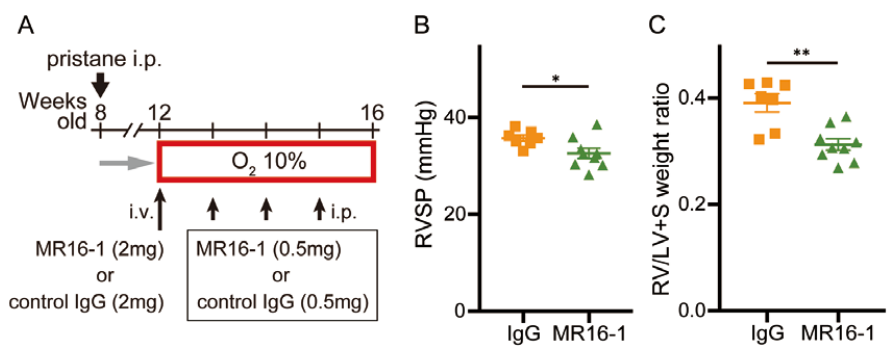

Figure 7. Interleukin (IL)-6 blockade ameliorated the pulmonary hypertension (PH) phenotype of the pristine/hypoxia (PriHx) mice. (A) Experimental protocol for determining the effect of antibody treatment on the PriHx mice. Anti-IL-6-R antibody (MR16-1) or control immunoglobulin G (IgG) was administered to C57BI/6J mice after administration of pristane. (B) Right ventricular systolic pressure (RVSP) of each group, $n=7-9$ in each group. (C) Right ventricle/left ventricle+septum (RV/LV+S) weight ratio, $n=7-9$ in each group. ${ }^{*} \mathrm{P}<0.05,{ }^{* *} \mathrm{P}<0.01$.

Background: Pulmonary arterial hypertension (PAH), particularly connective tissue disease-associated PAH (CTD-PAH), is a progressive disease and novel therapeutic agents based on the specific molecular pathogenesis are desired. In the pathogenesis of CTD-PAH, inflammation, immune cell abnormality, and fibrosis play important roles. However, the existing mouse pulmonary hypertension $(\mathrm{PH})$ models do not reflect these features enough. The relationship between inflammation and hypoxia is still unclear.

Methods and Results: Intraperitoneal administration of pristane, a kind of mineral oil, and exposure to chronic hypoxia were combined, and this model is referred to as pristane/hypoxia (PriHx) mice. Hemodynamic and histological analyses showed that the PriHx mice showed a more severe phenotype of PH than pristane or hypoxia alone. Immunohistological and flow cytometric analyses revealed infiltration of immune cells, including hemosiderin-laden macrophages and activated $\mathrm{CD}^{+}$helper $\mathrm{T}$ lymphocytes in the lungs of PriHx mice. Pristane administration exacerbated lung fibrosis and elevated the expression of fibrosis-related genes. Inflammationrelated genes such as $I l 6$ and $C x c l 2$ were also upregulated in the lungs of PriHx mice, and interleukin (IL)-6 blockade by monoclonal anti-IL-6 receptor antibody MR16-1 ameliorated PH of PriHx mice.

Conclusions: A PriHx model, a novel mouse model of PH reflecting the pathological features of CTD-PAH, was developed through a combination of pristane administration and exposure to chronic hypoxia.

Second Place in the Experimental Investigation Section

(Circ J 2020; 84: 1244-1253)6

\section{Magnesium Deficiency Causes Transcriptional Downregulation of Kir2.1 and Kv4.2 Channels in Cardiomyocytes Resulting in QT Interval Prolongation}

Toru Shimaoka, Yan Wang, Masaki Morishima, Shinji Miyamoto, Katsushige Ono

(Department of Pathophysiology (T.S., Y.W., M.M., K. O. ), Department of Cardiovascular Surgery (T.S., S.M.), Oita University School of Medicine, Yufu, Japan)

Background: Mechanisms for QT interval prolongation and cardiac arrhythmogenesis in hypomagnesemia are poorly understood. This study investigated the potential molecular mechanism for QT prolongation caused by magnesium $(\mathrm{Mg})$ deficiency in rats by using the patch clamp technique and molecular biology.

Methods and Results: Male Wistar rats were fed an Mg-free diet or a normal diet for up to 12 weeks. There was QT prolongation in the ECG of Mg-deficient rats, and cardiomyocytes from these rats showed prolongation of action potential duration. Electrophysiological studies showed that inward-rectifying $\mathrm{K}^{+}$current (IK1) and transient outward $\mathrm{K}^{+}$current ( $\mathrm{Ito}_{\mathrm{to}}$ ) were decreased in $\mathrm{Mg}$-deficient cardiomyocytes, and these findings were consistent with the downregulation of mRNA, as well as protein levels of Kir2.1 and Kv4.2. In Mg-deficient cardiomyocytes, transcription factors, GATA4 and NFAT, were upregulated, whereas CREB was downregulated. In contrast to $\mathrm{Mg}$ deficiency, cellular $\mathrm{Mg}^{2+}$ overload in cultured cardiomyocytes resulted in the upregulation of Kir2.1 and 


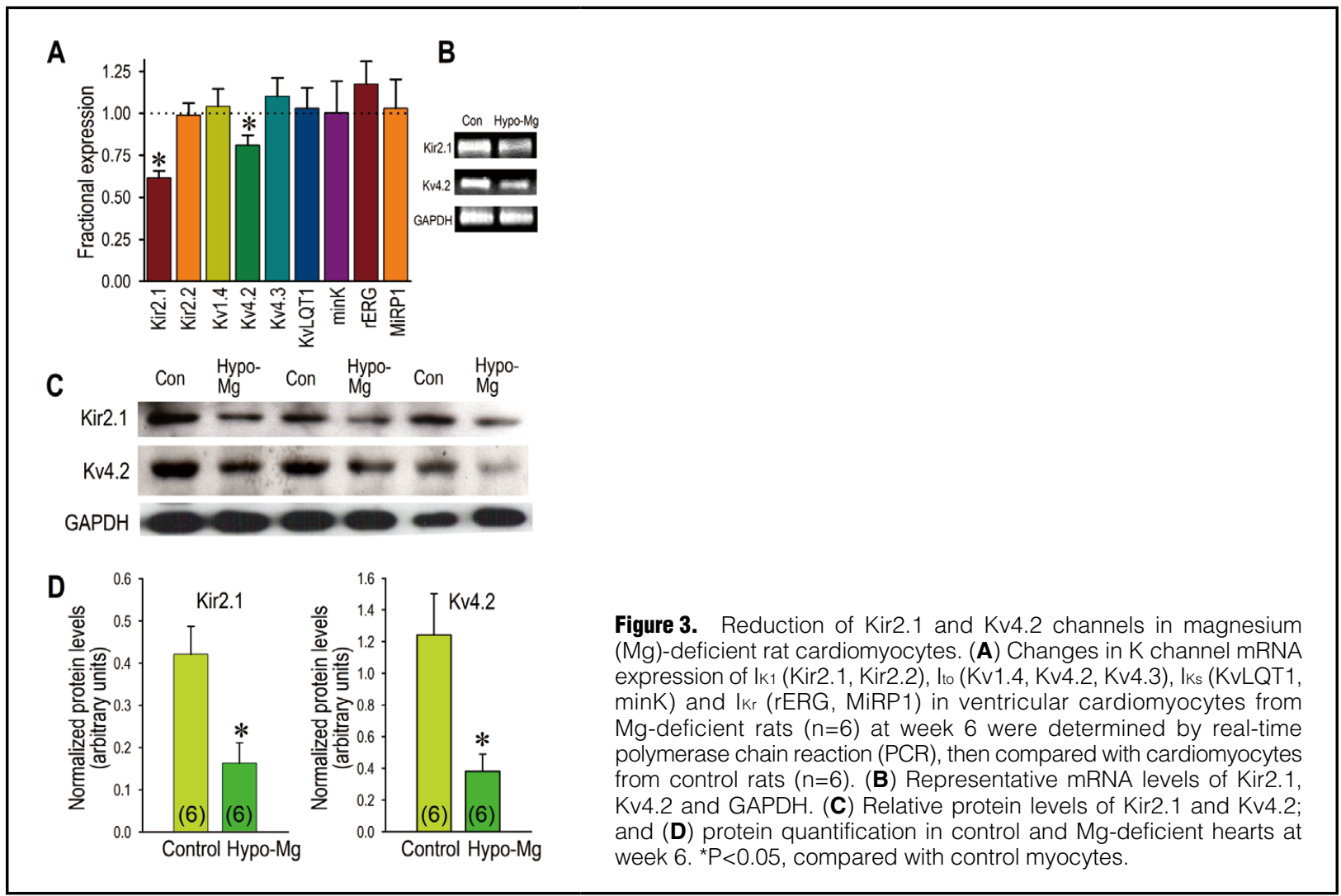

Kv4.2, which was accompanied by the downregulation of GATA4 and NFATc4, and the upregulation of CREB. Activation of NFAT and inhibition of CREB reduced Kv4.2-Ito, whereas Kir2.1-IK1 was reduced by CREB inhibition but not by NFTA activation.

Conclusions: Intracellular Mg deficiency downregulates IK1 and Ito in cardiomyocytes, and this is mediated by the transcription factors, NFAT and CREB. These results provide a novel mechanism for the long-term QT interval prolongation in hypomagnesemia.

\section{Comparison of Percutaneous Coronary Intervention, Coronary Artery} Bypass Grafting and Medical Therapy in Non-ST Elevation Acute Coronary Syndrome Patients With 3-Vessel Disease

Sida Jia, Ce Zhang, Lin Jiang, Lianjun Xu, Jian Tian, Xueyan Zhao, Xinxing Feng, Dong Wang, Yin Zhang, Kai Sun, Jingjing Xu, Ru Liu, Bo Xu, Wei Zhao, Rutai Hui, Runlin Gao, Zhan Gao, Jinqing Yuan, Lei Song

(Fu Wai Hospital, National Center for Cardiovascular Diseases, Peking Union Medical College \& Chinese Academy of Medical Sciences, Beijing, China)

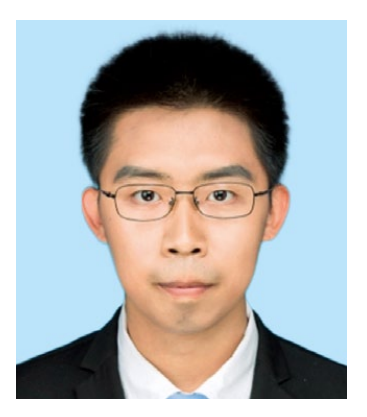

Background: The aim of this study is to compare the long-term prognosis of non-ST elevation acute coronary syndrome (NSTE-ACS) patients with 3-vessel disease (3VD) who underwent percutaneous coronary intervention (PCI), coronary artery bypass grafting (CABG) or medical therapy (MT).

Methods and Results: Overall, 3,928 NSTE-ACS patients with 3VD were consecutively enrolled from April 2004 to February 2011 at Fu Wai Hospital. Patients were followed up for a median of 7.5 years, and were divided into PCI, CABG or MT groups according to their treatment. Compared with patients undergoing PCI, CABG patients had lower rates of myocardial infarction (MI), unplanned revascularization, major adverse cardiovascular and cerebrovascular events (MACCE) and a higher rate of stroke (all $\mathrm{P}<0.05$ ). Compared with MT, PCI 
Table 3. Multivariable Cox Regression Analysis of CABG vs. PCl on Clinical Outcomes

\begin{tabular}{llrcc} 
& PCI & \multicolumn{3}{c}{ CABG } \\
All cause death & ref & HR (95\% Cl) & P value \\
Cardiac death & ref & $0.910(0.580-1.429)$ & 0.683 \\
Revascularization & ref & $0.445(0.226-0.876)$ & 0.019 \\
MI & ref & $0.330(0.172-0.635)$ & 0.001 \\
Stroke & ref & $0.585(0.269-1.274)$ & 0.177 \\
MACCE & ref & & $1.117(0.636-1.962)$ & 0.701 \\
Death/Ml/stroke & ref & & $0.719(0.532-0.972)$ & 0.032 \\
& & & $0.884(0.636-1.229)$ & 0.463 \\
\end{tabular}

Adjusted variables: left main disease, age, gender, history of diabetes, history of Ml, history of peripheral artery disease, history of chronic kidney disease, clinical presentation (acute MI, unstable angina), LVEF, serum creatinine, creatinine clearance, preprocedural SYNTAX score, medication at discharge (ACEI, ARBs, aspirin, clopidogrel, statins, nitrates, calcium channel blockers), hemoglobin, assigned treatment. Cl, confidence interval; HR, hazard ratio; ref, reference. Other abbreviations as in Tables 1,2.

\section{A. All-cause death}

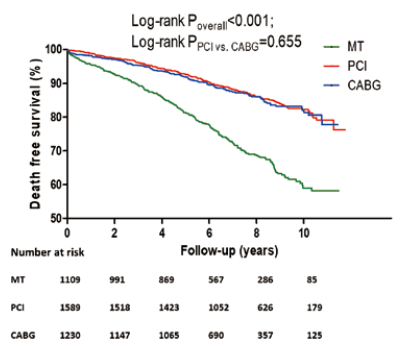

Cass $\quad 1230 \quad 12197 \quad 1005 \quad 690 \quad 357 \quad 125$ C. Revascularization
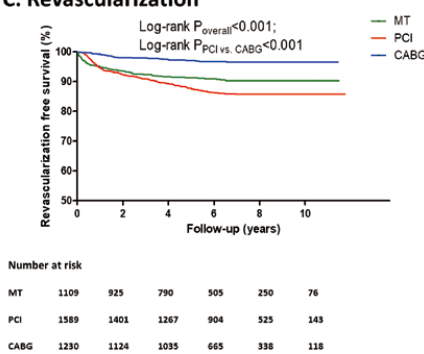

CABG $\quad 1230 \quad 1124 \quad 1035 \quad 663 \quad 398 \quad 128$

E. Stroke Log-rank $P_{\text {overal }}<0.001 ;$
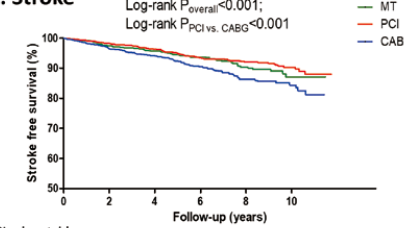

Number at rist

$\begin{array}{lllllll}\text { MT } & 1109 & 967 & 834 & 535 & 261 & 76\end{array}$

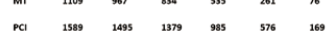

CABG $\quad{ }^{1230} \quad{ }^{1107} \quad{ }^{1007}$

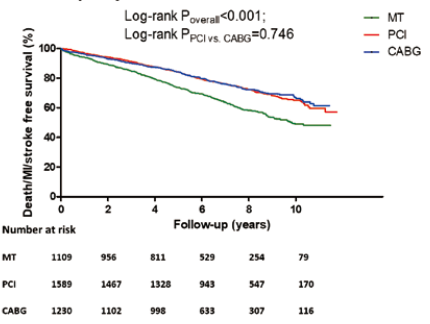

\section{a}

\section{B. Cardiac death}
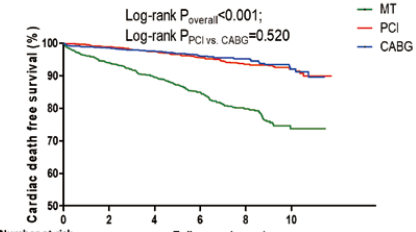

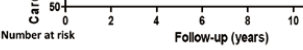

$\begin{array}{lllllll}\text { MT } & 1109 & 991 & 870 & 567 & 286 & 85\end{array}$

$\begin{array}{llllllll}1589 & 1519 & 1023 & 1052 & 626 & 179\end{array}$

$\begin{array}{lllllll}\text { CABG } \quad{ }^{1230} & 1297 & 1065 & 690 & 357 & 125\end{array}$

D. MI
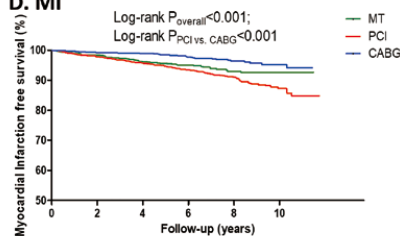

Number at risk

$\begin{array}{lllllll}\text { MT } & 1109 & 976 & 837 & 538 & 265 & 82\end{array}$

$\begin{array}{lllllll}\mathrm{PCl} & 1589 & 1499 & 1866 & 981 & 561 & 160\end{array}$

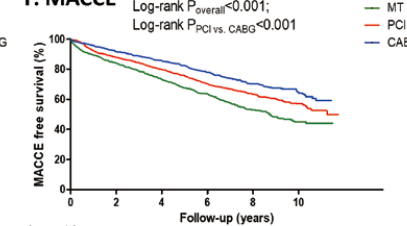

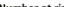

$\begin{array}{lllllll}\text { MT } \quad 1109 & 900 & 741 & 467 & 222 & 66\end{array}$

$\begin{array}{lllllll}\mathrm{PCl} & 1589 & 1370 & 1206 & 820 & 452 & 125\end{array}$

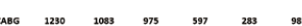

Figure 2. Kaplan-Meier survival curves on long-term clinical endpoints in percutaneous coronary intervention (PCI), coronary artery bypass grafting (CABG) and medical therapy (MT) groups for 3-vessel disease (3VD) patients presenting with non-ST elevation acute coronary syndrome (NSTE-ACS). (A) All-cause death; (B) cardiac death; (C) unplanned revascularization; (D) myocardial infarction; (E) stroke; (F) major adverse cardiac and cerebrovascular event (MACCE); (G) death/Ml/stroke. 
and $\mathrm{CABG}$ had lower incidences of all adverse outcomes (all $\mathrm{P}<0.05$ ), except for a similar rate of stroke between PCI and MT. Kaplan-Meier analysis showed similar results. After adjusting for confounders, CABG was independently associated with a lower risk of cardiac death, revascularization and MACCE compared with PCI (all $\mathrm{P}<0.05$ ). Compared with MT, PCI reduced long-term risk of death, whereas CABG reduced long-term risk of death, revascularization and MACCE events (all $\mathrm{P}<0.05$ ).

Conclusions: In NSTE-ACS patients with $3 \mathrm{VD}, \mathrm{CABG}$ is independently associated with a lower risk of longterm cardiac death, revascularization and MACCE compared with PCI. Patients who received MT alone had the highest risk of long-term MACCE.

Awards will be presented to the 7 research groups during the $85^{\text {th }}$ Annual Scientific Meeting of the Japanese Circulation Society, and will also be announced on the Society website. We look forward to receiving manuscripts with high scientific impact for publication in Circulation Journal in 2021.

Toshihisa Anzai, MD, PhD

Editor-in-Chief

Circulation Journal

\section{References:}

1. Ito H, Wakatsuki T, Yamaguchi K, Fukuda D, Kawabata Y, Matsuura T, et al. Atherosclerotic coronary plaque is associated with adventitial vasa vasorum and local inflammation in adjacent epicardial adipose tissue in fresh cadavers. Circ J 2020; 84: 769-775.

2. Yanase M, Iwasaki K, Watanabe T, Seguchi O, Nakajima S, Kuroda K, et al. Effect of therapeutic modification on outcomes in heart transplantation over the past two decades: A single-center experience in Japan. Circ J 2020; 84: 965-974.

3. Sato T, Calderon RJC, Klas B, Pedrizzetti G, Banerjee A. Simultaneous volumetric and functional assessment of the right ventricle in hypoplastic left heart syndrome after fontan palliation, utilizing 3-dimensional speckle-tracking echocardiography. Circ J 2020; 84: $235-244$.

4. Arase H, Yamada S, Tanaka S, Tokumoto M, Tsuruya K, Nakano T, et al. Association between plasma intact parathyroid hormone levels and the prevalence of atrial fibrillation in patients with chronic kidney disease: The Fukuoka kidney disease registry study. Circ $J$ 2020; 84: 1105-1111.

5. Mori H, Ishibashi T, Inagaki T, Okazawa M, Masaki T, Asano R, et al. Pristane/hypoxia (PriHx) mouse as a novel model of pulmonary hypertension reflecting inflammation and fibrosis. Circ J 2020; 84: 1163-1172.

6. Shimaoka T, Wang Y, Morishima M, Miyamoto S, Ono K. Magnesium deficiency causes transcriptional downregulation of Kir2.1 and Kv4.2 channels in cardiomyocytes resulting in QT interval prolongation. Circ J 2020; 84: 1244-1253.

7. Jia S, Zhang C, Jiang L, Xu L, Tian J, Zhao X, et al. Comparison of percutaneous coronary intervention, coronary artery bypass grafting and medical therapy in non-ST elevation acute coronary syndrome patients with 3-vessel disease. Circ J 2020; 84: 1718 -1727. 